\title{
In-stent restenosis assessed with frequency domain optical coherence tomography shows smooth coronary arterial healing process in second-generation drug-eluting stents
}

Takashi Kajiya ${ }^{1}$, MD, Hiroshi Yamaguchi ${ }^{2}$, MD, Junichiro Takaoka ${ }^{1}$, MD, Kengo Fukunaga ${ }^{1}$, MD, Ryoichi Arima ${ }^{1}$, MD, Akihiro Miyamura ${ }^{1}$, MD, Toshiko Ninomiya ${ }^{1}$, MD, Nobuhiko Atsuchi ${ }^{1}$, MD, PhD, Yoshihiko Atsuchi ${ }^{1}, \mathrm{MD}, \mathrm{PhD}$, Mitsuyasu Terashima ${ }^{3}$, MD, PhD, Hideaki Kaneda ${ }^{4}$, MD, PhD, Mitsuru Ohishi ${ }^{5}$, MD, PhD

INTRODUCTION The pathophysiology and mechanism of in-stent restenosis (ISR) after implantation of second-generation drug-eluting stents (DESs) are not fully clear. We compared the morphological characteristics of ISR between first- and second-generation DESs using frequency domain optical coherence tomography (OCT).

METHODS Patients who underwent follow-up coronary angiography (CAG) after first-generation (CYPHER ${ }^{\text {TM }}$ and TAXUS ${ }^{\text {TM }}$ ) and second-generation (Nobori ${ }^{\circledR}$, PROMUS Element ${ }^{\mathrm{TM}}$, Resolute Integrity and XIENCE) DES implantations were examined. ISR was defined as lesions of over $50 \%$ diameter stenosis at follow-up CAG. Frequency domain OCT was performed at the time of revascularisation of ISR. Tissue morphology was assessed at minimum lumen area. OCT images of DESS at both early ( $\leq 1$ year) and late ( $>1$ year) phase follow-up were compared.

RESULTS On qualitative OCT assessment, the ratios of homogeneous, layered, heterogeneous without-attenuation and heterogeneous with-attenuation morphologies were $57.1 \%, 17.1 \%, 20.0 \%$ and $5.7 \%$, respectively, for second-generation DES ISR $(n=35)$, and $16.7 \%, 25.0 \%, 25.0 \%$ and 33.3\%, respectively, for first-generation DES ISR $(n=36)$. At late phase follow-up, homogeneous morphology was significantly more common for second-generation DES ISR compared to firstgeneration DES ISR (first-generation: $8.0 \%$ vs. second-generation: $50.0 \%$; $<0.01$ ) while heterogeneous with-attenuation morphology was significantly more common for first-generation DES ISR (first-generation: $44.0 \%$ vs. second-generation: $5.6 \% ; p<0.01)$.

CONCLUSION Homogeneous tissue morphology was more frequently found for second-generation than first-generation DES ISR, especially in the late phase. This suggested that neointimal hyperplasia was the main mechanism in secondgeneration DES ISR, and that the neointima was stabilised, much like in bare metal stent implantation.

Keywords: DES, drug-eluting stent, optical coherence tomography

\section{INTRODUCTION}

In-stent restenosis (ISR) is not commonly seen with secondgeneration drug-eluting stents (DESs), but still occurs occasionally. Improvement of stent platforms and polymer coating are some reasons for the lower number of cases of ISR following second-generation DES implantation when compared with firstgeneration DES implantation. However, the pathophysiology and mechanism of ISR following second-generation DES implantation have not been fully clarified.

Frequency domain optical coherence tomography (OCT), which is now in use, has superior resolution than time-domain OCT. We assessed the tissue morphology of second-generation DES ISR using frequency domain OCT and compared the findings to those of first-generation DES ISR.

\section{METHODS}

We conducted a retrospective study of 2,074 lesions that were implanted with DESs at a single institute from April
2008 to December 2014. From April 2008 to January 2011, first-generation DESs were implanted for 805 lesions and from January 2011 to December 2014, second-generation DESs were implanted for 1,269 lesions at our hospital. The CYPHER ${ }^{\text {TM }}$ sirolimus-eluting coronary stent (Cordis, Miami Lakes, FL, USA) and TAXUS ${ }^{\text {TM }}$ paclitaxel-eluting coronary stent system (Boston Scientific, Natick, MA, USA) were defined as first-generation DESs. The Nobori ${ }^{\circledR}$ biolimus-eluting stent (Terumo, Tokyo, Japan), PROMUS Element ${ }^{\mathrm{TM}}$ everolimus-eluting coronary stent system (Boston Scientific, Natick, MA, USA), Resolute Integrity zotarolimus-eluting coronary stent system (Medtronic, Santa Rosa, CA, USA) and XIENCE everolimus-eluting coronary stent system (Abbott Vascular, Santa Clara, CA, USA) were defined as second-generation DESs.

The first scheduled follow-up coronary angiography (CAG) was performed at 6-9 months after percutaneous coronary intervention and the second CAG at 18-24 months after percutaneous coronary intervention. All patients had been taking

\footnotetext{
${ }^{1}$ Department of Cardiology, Tenyoukai Central Hospital, ${ }^{2}$ Yamaguchi Hiroshi Clinic, Kagoshima, ${ }^{3}$ Toyohashi Heart Center, Toyohashi, ${ }^{4}$ Okinaka Memorial Institute for Medical Research, Tokyo, ${ }^{5}$ Department of Cardiovascular Medicine and Hypertension, Kagoshima University, Kagoshima, Japan

Correspondence: Dr Takashi Kajiya, Senior Consultant, Department of Cardiology, Tenyoukai Central Hospital, Izumi-cho 6-7, Kagoshima-city, 892-0611, Japan. t_kajiya@hotmail.com
} 
aspirin (dose $100 \mathrm{mg} /$ day) with clopidogrel (dose $75 \mathrm{mg} /$ day) or prasugrel (dose $3.5 \mathrm{mg} /$ day) for at least one year. In cases where patients complained of ischaemic symptoms, follow-up CAG was performed earlier than the scheduled date. ISR was defined as lesions more than $50 \%$ diameter stenosis with myocardial ischaemia at follow-up CAG. All patients with ISR were categorised as having early ( $\leq 1$ year) or late ( $>1$ year) phase ISR.

Frequency domain OCT was performed at the time of revascularisation for ISR. This imaging procedure was performed using an optical frequency domain imaging system (Terumo Corporation, Tokyo, Japan) or an M2 OCT system (LightLab Imaging, Westford, MA, USA). The optical frequency domain imaging used a non-occlusive technique at an image acquisition rate of 158 frames per second during an automated pullback at a speed of $40 \mathrm{~mm} / \mathrm{s}$. The pullback was performed during continuous intracoronary injection of contrast medium through the $\geq 6$-French guiding catheter, using an injection pump at a flow rate of $3.0 \mathrm{~mL} / \mathrm{s}$ for the left coronary artery and $2.5 \mathrm{~mL} / \mathrm{s}$ for the right coronary artery for four seconds. ${ }^{(1)}$ The M2 OCT system was used, in accordance with previous reports. ${ }^{(2)}$

Patients with stent fractures, stent recoils or in-stent calcified nodules were excluded from analysis. As previously reported, ${ }^{(3,4)}$ to evaluate the morphological appearance of restenotic tissue, the pattern of restenotic tissue in cross-sectional images at minimal lumen area was categorised into three groups: (a) homogeneous pattern; (b) layered; and (c) heterogeneous. Homogeneous pattern meant a uniformly signal-rich appearance. Layered pattern meant a signal-poor appearance, with a high-signal band adjacent to the luminal surface. Heterogeneous pattern meant a mainly signalpoor appearance, with islands of various signal regions, and was further divided into two patterns with or without attenuation. Next, the frequency domain OCT images of second-generation DES ISR of the early ( $\leq 1$ year) and late ( $>1$ year) phases were compared with those of first-generation DES ISR. All images were analysed by independent observers who were masked to the patients' clinical presentation and lesion characteristics.

Discrete data was presented as frequencies and percentages, and continuous variables were presented as mean \pm standard deviation. Continuous variables were compared using $t$-test, and categorical variables were compared using either chi-square test or Fisher's exact test, as appropriate. A p-value $<0.05$ was considered to be statistically significant.

\section{RESULTS}

The incidence of ISR among patients for whom second-generation DESs were implanted was significantly lower than that for first-generation DESs (second-generation: 51/1,269 [4.0\%] vs. first-generation: 77/805 [9.6\%]; $p<0.01$ ). Baseline clinical characteristics of patients with ISR following first- and secondgeneration DES implantation are shown in Table I.

Of 51 second-generation DES patients, 44 patients with second-generation DES ISR were evaluated using frequency domain OCT. During qualitative frequency domain OCT assessment, nine patients were excluded because stent fracture or recoil with/without calcified nodules was considered to
Table I. Baseline clinical characteristics of patients with in-stent restenosis following DES implantation.

\begin{tabular}{|lll|}
\hline \multirow{2}{*}{ Variable } & \multicolumn{2}{c|}{ No. (\%)/mean \pm SD } \\
\cline { 2 - 3 } & $\begin{array}{l}\text { First-generation } \\
\text { DES }(\mathbf{n}=\mathbf{3 6})\end{array}$ & $\begin{array}{l}\text { Second-generation } \\
\text { DES }(\mathbf{n}=\mathbf{3 5})\end{array}$ \\
\hline Age (yr) & $72.2 \pm 11.0$ & $70.3 \pm 13.5$ \\
\hline Male gender & $27(75.0)$ & $29(82.9)$ \\
\hline Comorbidity & & \\
\hline Hypertension & $30(83.3)$ & $25(71.4)$ \\
\hline Diabetes mellitus & $20(55.6)$ & $24(68.6)$ \\
\hline Dyslipidaemia & $19(52.8)$ & $21(60.0)$ \\
\hline Smoking & $8(22.2)$ & $8(22.9)$ \\
\hline Family history & $0(0)$ & $0(0)$ \\
\hline Haemodialysis & $6(16.7)$ & $5(14.3)$ \\
\hline Stent size (mm) & $2.8 \pm 0.3$ & $2.8 \pm 0.4$ \\
\hline
\end{tabular}

DES: drug-eluting stent; SD: standard deviation

Table II. Optical coherence tomography findings of patients with in-stent restenosis following DES implantation.

\begin{tabular}{|c|c|c|c|}
\hline \multirow[t]{2}{*}{ Variable } & \multicolumn{2}{|c|}{ No. (\%) } & \multirow{2}{*}{ p-value } \\
\hline & $\begin{array}{l}\text { First-generation } \\
\text { DES }(n=36)\end{array}$ & $\begin{array}{l}\text { Second-generation } \\
\text { DES }(n=35)\end{array}$ & \\
\hline \multicolumn{4}{|l|}{ ISR morphology } \\
\hline Homogeneous & $6(16.7)$ & $20(57.1)$ & $<0.01$ \\
\hline Layered & $9(25.0)$ & $6(17.1)$ & 0.5 \\
\hline \multicolumn{4}{|l|}{ Heterogeneous } \\
\hline $\begin{array}{l}\text { Without } \\
\text { attenuation }\end{array}$ & $9(25.0)$ & $7(20.0)$ & 0.5 \\
\hline $\begin{array}{l}\text { With } \\
\text { attenuation }\end{array}$ & $12(33.3)$ & $2(5.7)$ & $<0.01$ \\
\hline Min & 1.9 & 1.6 & 0.6 \\
\hline$\%$ plaque area & 74.3 & 75.2 & 0.6 \\
\hline
\end{tabular}

DES: drug-eluting stent; ISR: in-stent restenosis; Min: minimum plaque area $\left(\mathrm{mm}^{2}\right)$

be the cause of ISR. Among the 35 remaining patients with second-generation DES ISR, $14.3 \%(n=5)$ of patients had haemodialysis and mean creatinine was high at $3.3 \pm 4.5 \mathrm{mg} / \mathrm{dL}$. The corresponding number of patients with first-generation DES ISR who were finally included in the analysis was 36 . There was no significant difference in plaque volume between first- and second-generation DESs (Table II) and stent struts were visually well covered in all patients. On qualitative OCT assessment, the ratios of homogeneous, layered, heterogeneous withoutattenuation and heterogeneous with-attenuation morphologies were $57.1 \% 17.1 \%, 20.0 \%$ and $5.7 \%$, respectively, for secondgeneration DES ISR $(\mathrm{n}=35)$, and $16.7 \%, 25.0 \%, 25.0 \%$ and $33.3 \%$, respectively, for first-generation DES ISR $(\mathrm{n}=36)$. When compared with first-generation DES ISR $(n=36)$, the incidence of a homogeneous pattern was significantly higher in second-generation DES ISR (second-generation: $57.1 \%$ vs. first-generation: $16.7 \% ; p<0.01)$. On the other hand, the heterogeneous pattern with attenuation was more frequently seen in first-generation DES ISR (second-generation: $5.7 \%$ vs. first-generation: $33.3 \% ; p<0.01$ ). 
Table III. Morphologies of patients with in-stent restenosis following DES implantation in the early phase.

\begin{tabular}{|llll|}
\hline Morphology & \multicolumn{2}{c}{ No. (\%) } & p-value \\
\cline { 2 - 3 } & $\begin{array}{l}\text { First-generation } \\
\text { DES }(\mathbf{n}=\mathbf{1 1})\end{array}$ & $\begin{array}{l}\text { Second-generation } \\
\text { DES }(\mathbf{n}=\mathbf{1 7})\end{array}$ & \\
\hline Homogeneous & $4(36.4)$ & $11(64.7)$ & 0.14 \\
\hline Layered & $3(27.3)$ & $1(5.9)$ & 0.15 \\
\hline $\begin{array}{l}\text { Heterogeneous } \\
\text { Without }\end{array}$ & $3(27.3)$ & $4(23.5)$ & 0.5 \\
\hline $\begin{array}{l}\text { Wttenuation } \\
\text { With }\end{array}$ & $1(9.1)$ & $1(5.9)$ & 0.6 \\
\hline
\end{tabular}

DES: drug-eluting stent

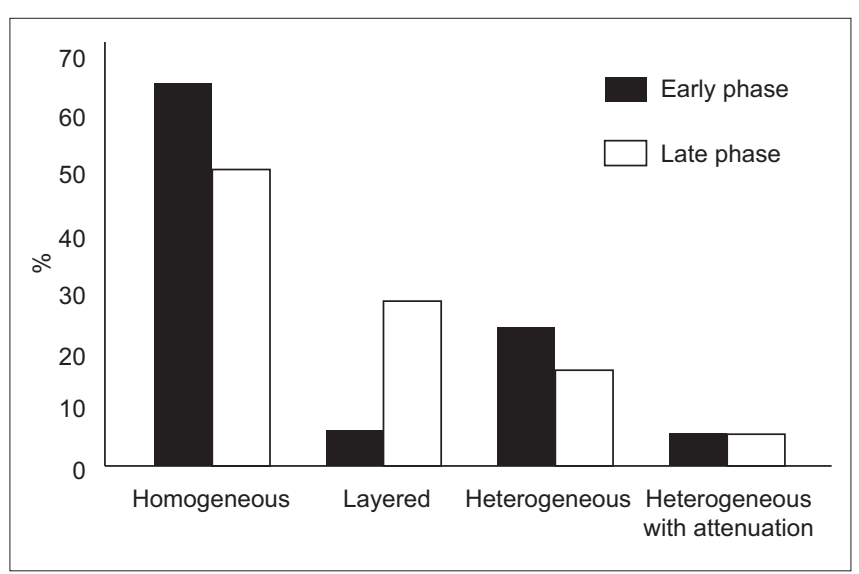

Fig. 1 Chart shows the proportion of in-stent restenosis following second generation drug-eluting stent implantation in the early ( $\leq 1$ year) and late (>1 year) phases.

All patients with ISR were divided into two groups of early phase ( $\leq 1$ year) and late phase ( $>1$ year). The mean time period of DES ISR in the early phase was $6.3 \pm 2.3$ months and that of the late phase was $20.5 \pm 8.5$ months. In the early phase, there was a trend for the homogeneous pattern to be higher in second-generation DES ISR, although this was not statistically significant (second-generation: $64.7 \%$ vs. first-generation: $36.4 \%$; $\mathrm{p}=0.14$ ) (Table III). In the late phase, the homogeneous pattern was significantly higher in second-generation DES ISR (secondgeneration: $50.0 \%$ vs. first-generation: $8.0 \%$; $<<0.01$ ) (Table IV). On the other hand, the heterogeneous pattern with attenuation was significantly higher in first-generation DES ISR in the late phase (second-generation: $5.6 \%$ vs. first-generation: $44.0 \%$; $p<0.01$ ). For second-generation DES ISR, the homogeneous morphology was the main mechanism in both the early and late phases, with the proportion of layered morphology increasing in the late phase (Fig. 1). The distribution over time of morphologies among patients with second-generation DES ISR in the late phase is shown in Fig. 2.

\section{DISCUSSION}

We investigated the morphology of second-generation DES ISR using high-resolution frequency domain OCT. The homogeneous pattern was found in both early and late phase ISR. According to pathologic evaluations of human ISR tissue, a cell-rich pattern
Table IV. Morphologies of patients with in-stent restenosis following DES implantation in the late phase.

\begin{tabular}{|llll|}
\hline Morphology & \multicolumn{2}{c}{ No. (\%) } & p-value \\
\cline { 2 - 3 } & $\begin{array}{l}\text { First-generation } \\
\text { DES }(\mathbf{n}=\mathbf{2 5})\end{array}$ & $\begin{array}{l}\text { Second-generation } \\
\text { DES (n= 18) }\end{array}$ & \\
\hline Homogeneous & $2(8.0)$ & $9(50.0)$ & $<0.01$ \\
\hline Layered & $6(24.0)$ & $5(27.8)$ & 0.5 \\
\hline Heterogeneous & & $3(16.7)$ & 0.4 \\
\hline $\begin{array}{l}\text { Without } \\
\text { attenuation }\end{array}$ & $6(24.0)$ & $1(5.6)$ & $<0.01$ \\
\hline $\begin{array}{l}\text { With } \\
\text { attenuation }\end{array}$ & $11(44.0)$ & & \\
\hline
\end{tabular}

DES: drug-eluting stent

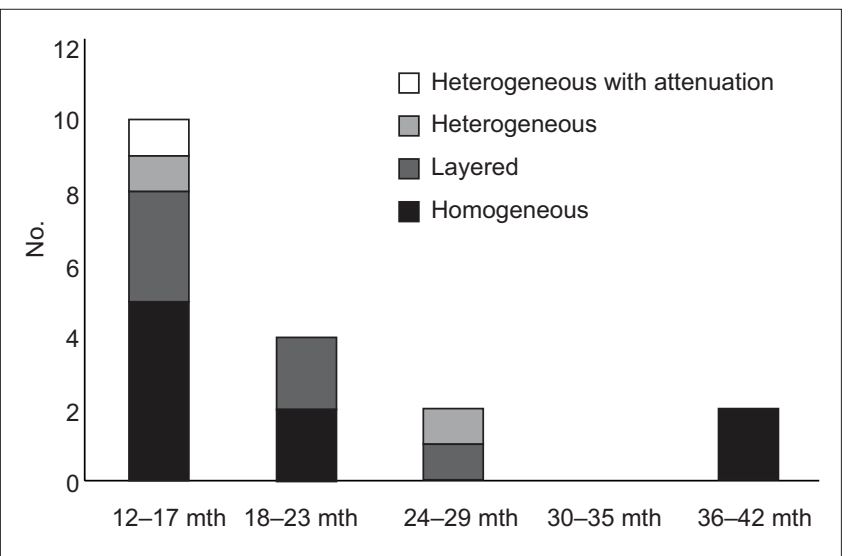

Fig. 2 Chart shows the distribution of morphologies among secondgeneration drug-eluting stent patients with in-stent restenosis in the late phase ( $>1$ year), according to time period.

(homogeneous on OCT) likely comprised smooth muscle cells with collagen fibres, whereas a cell-poor pattern (heterogeneous on OCT) likely included a lipid pool with proteoglycans, cell matrix, fibrinoids and organised thrombi. ${ }^{(5)}$ Therefore, neointimal tissue was considered as the main cause of second-generation DES ISR both in the early ( $\leq 1$ year) and late ( $>1$ year) phases.

Previous studies have shown that most cases of ISR involving bare metal stents (BMSs) have a homogeneous pattern (91\%) in the early phase. ${ }^{(6)}$ This implies that the arterial healing process starts smoothly in the early phase following BMS implantation. The American College of Cardiology/American Heart Association guideline mentioned that dual antiplatelet therapy should be continued for at least six months after the implantation of secondgeneration DESs. ${ }^{(7)}$ Currently, dual antiplatelet therapy is usually stopped at one month after BMS implantation. This is because after one month, BMS struts are fully covered by neointimal tissue and the possibility of stent thrombosis becomes low. During the time when first-generation DESs were used, stent thrombosis was a major concern for interventional cardiologists. Some stent struts were not covered by neointima even in the late phase, resulting in thrombus formation at uncovered struts. Imai et al reported that in lesions with peri-stent contrast staining, the incidence of very late stent thrombosis at four years after the index follow-up CAG was significantly higher than in lesions without it. ${ }^{(8)}$ Subsequently, long-term dual antiplatelet therapy was recommended for patients 
after first-generation DES stenting and the second-generation DES was developed.

Some studies have shown that the stent thrombosis rate in second-generation DES implantation was lower than that for BMS implantation. ${ }^{(9,10)}$ Another study found that stent strut coverage was good a few months after second-generation DES implantation. ${ }^{(11}$ It has also been reported that a shortening of the dual antiplatelet therapy period might be possible for patients who are implanted with second-generation DESs. ${ }^{(12)}$ Our study supports previous data that the arterial healing process is smooth ${ }^{(13)}$ and that stent struts are mainly covered with neointima in both early and late phase ISR when second-generation DESs are used. As a result, stent thrombosis can be expected to be rare in patients with secondgeneration DES implantation.

Although the histopathology of heterogeneous structures has remained unclear, our OCT findings indicated that heterogeneous intima with attenuation can be considered to represent neoatherosclerosis. A previous study suggested that the occurrence of neoatherosclerosis following stent implantation has not been ruled out even with the use of second-generation DESs. ${ }^{(5)}$ Jinnouchi et al reported that the presence of neoatherosclerosis was the main cause of late ISR following second-generation DES implantation, and that it increases over time. ${ }^{(14)}$ Further studies are required to clarify the manner in preventing and treating in-stent neoatherosclerosis. ${ }^{(14)}$

On the other hand, layered structures consist of a homogeneous inner layer with a high or low backscattered outer layer. Such lesions might include a certain amount of vascular smooth muscle cells in their inner layer. The paclitaxel-coated balloon therefore is effective for these types of layered neointima. ${ }^{(15)}$

In the present study, the homogeneous and heterogeneous patterns were nearly fixed in the early and late phase secondgeneration DES ISR. However, in the late phase, the heterogeneous pattern with attenuation decreased in second-generation DES ISR. This suggests that inflammatory processes and/or thrombus formation decrease in the late phase following second-generation DES implantation.

This study had limitations related to its study design and methods of data collection. It was a retrospective non-randomised study, and hence we could not eliminate all confounding factors and biases. There was a possibility of substantial selection bias with regard to patient selection, as well as the experience and dedication of the operators. Finally, this study included patients with endstage renal failure, which is associated with different pathological processes compared with patients with non-end-stage renal failure. The latter group of patients may have different OCT findings.

In conclusion, homogeneous tissue morphology on frequency domain OCT was more frequently found in second-generation
DES ISR than in first-generation DES ISR, both in the early ( $\leq 1$ year) and late ( $>1$ year) phases. This finding suggests that neointimal hyperplasia is the main mechanism in secondgeneration DES ISR and that the arterial healing process is smooth, much like in BMS implantation.

\section{REFERENCES}

1. Fukunaga K, Kajiya T, Takaoka J, et al. Smooth arterial healing after paclitaxelcoated balloon angioplasty for in-stent restenosis assessed with optical frequency domain imaging. Asialntervention 2016; 2:44-7.

2. Gonzalo N, Serruys PW, Okamura T, et al. Optical coherence tomography patterns of stent restenosis. Am Heart J 2009; 158:284-93.

3. Arikawa R, Yamaguchi H, Takaoka J, et al. Simple balloon dilation for drugeluting in-stent restenosis: an optical coherent tomography analysis. Cardiovasc Revasc Med 2015; 16:27-31.

4. Yamaguchi H, Arikawa R, Takaoka J, et al. Association of morphologic characteristics on optical coherence tomography and angiographic progression patterns of late restenosis after drug-eluting stent implantation. Cardiovasc Revasc Med 2015; 16:32-5.

5. Nakano M, Virmani R. Histopathology of vascular response to drug-eluting stents: an insight from human autopsy into daily practice. Cardiovasc Interv Ther 2015; 30:1-11.

6. Goto K, Takebayashi H, Kihara Y, et al. Appearance of neointima according to stent type and restenotic phase: analysis by optical coherence tomography. Eurolntervention 2013; 9:601-7.

7. Levine GN, Bates ER, Bittl JA, et al. 2016 ACC/AHA guideline focused update on duration of dual antiplatelet therapy in patients with coronary artery disease: a report of the American College of Cardiology/American Heart Association Task Force on Clinical Practice Guidelines: an update of the 2011 ACCF/AHA/SCAI guideline for percutaneous coronary intervention, 2011 ACCF/AHA guideline for coronary artery bypass graft surgery, 2012 ACC/AHA/ACP/AATS/PCNA/SCAI/ STS guideline for the diagnosis and management of patients with stable ischemic heart disease, 2013 ACCF/AHA guideline for the management of ST-elevation myocardial infarction, $2014 \mathrm{AHA}$ ACC guideline for the management of patients with non-ST-elevation acute coronary syndromes, and 2014 ACC/AHA guideline on perioperative cardiovascular evaluation and management of patients undergoing noncardiac surgery. Circulation 2016; 134:e123-55.

8. Imai M, Kimura T, Morimoto T, et al. Impact of angiographic peri-stent contrast staining (PSS) on late adverse events after sirolimus-eluting stent implantation: an observation from the multicenter j-Cypher registry PSS substudy. Cardiovasc Interv Ther 2014; 29:226-36.

9. Kereiakes DJ, Yeh RW, Massaro JM, et al; DAPT Study Investigators. Stent thrombosis in drug-eluting or bare-metal stents in patients receiving dual antiplatelet therapy. JACC Cardiovasc Interv 2015; 8:1552-62.

10. Philip F, Stewart S, Southard JA. Very late stent thrombosis with second generation drug eluting stents compared to bare metal stents: network metaanalysis of randomized primary percutaneous coronary intervention trials. Catheter Cardiovasc Interv 2016; 88:38-48.

11. Kim S, Kim JS, Shin DH, et al. Comparison of early strut coverage between zotarolimus- and everolimus-eluting stents using optical coherence tomography. Am J Cardiol 2013; 111:1-5.

12. Généreux $P$, Rutledge DR, Palmerini $T$, et al. Stent thrombosis and dual antiplatelet therapy interruption with everolimus-eluting stents: insights from the Xience V Coronary Stent System trials. Circ Cardiovasc Interv 2015; 8:e001362.

13. Magalhaes MA, Minha S, Chen F, et al. Clinical presentation and outcomes of coronary in-stent restenosis across 3-stent generations. Circ Cardiovasc Interv 2014; 7:768-76.

14. Jinnouchi H, Kuramitsu S, Shinozaki T, et al. Difference of tissue characteristics between early and late restenosis after second-generation drug-eluting stents implantation: an optical coherence tomography study. Circ J 2017 Jan 27. [Epub ahead of print]

15. Tada T, Kadota K, Hosogi S, et al. Association between tissue characteristics evaluated with optical coherence tomography and mid-term results after paclitaxel-coated balloon dilatation for in-stent restenosis lesions: a comparison with plain old balloon angioplasty. Eur Heart J Cardiovasc Imaging 2014; 15:307-15 